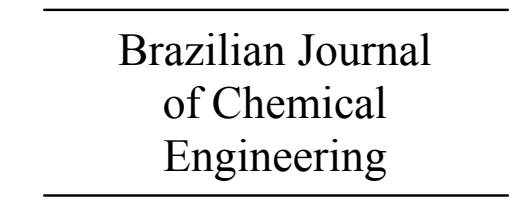

ISSN 0104-6632

Printed in Brazil

www.abeq.org.br/bjche

Vol. 29, No. 03, pp. 511 - 518, July - September, 2012

\title{
SECOND LAW ANALYSIS FOR FREE CONVECTION IN NON-NEWTONIAN FLUIDS OVER A HORIZONTAL PLATE EMBEDDED IN A POROUS MEDIUM: (PRESCRIBED HEAT FLUX)
}

\author{
W. A. Khan ${ }^{1 *}$ and R. S. R. Gorla ${ }^{2}$ \\ ${ }^{1}$ Department of Engineering Sciences, PN Engineering College, Phone: + 092-3218929467, \\ National University of Sciences and Technology, PNS Jauhar, Karachi 75350, Pakistan. \\ E-mail: wkhan_2000@yahoo.com \\ ${ }^{2}$ Department of Mechanical Engineering, Cleveland State University, Cleveland, Ohio, USA. \\ (Submitted: May 27, 2010 ; Revised: August 30, 2011 ; Accepted: February 26, 2012)
}

\begin{abstract}
Second law characteristics of heat transfer and fluid flow due to free convection of non-Newtonian fluids over a horizontal plate with prescribed surface heat flux in a porous medium are analyzed. Velocity and temperature fields are obtained numerically using an implicit finite difference method under the similarity assumption and these results are used to compute the entropy generation rate Ns, irreversibility ratio $\varphi$ and the Bejan number Be for both Newtonian and non-Newtonian fluids. The effects of power-law index $\mathrm{n}$, heat flux variation parameter $\lambda$, and modified duty parameter, $G$ on the dimensionless entropy generation rate Ns, and the Bejan number Be are investigated and presented graphically.

Keywords: Second law; Free convection; Non-Newtonian fluids; Porous medium; Plate.
\end{abstract}

\section{INTRODUCTION}

The second law analysis of a non-Newtonian fluid over a horizontal surface has many significant applications in thermal engineering and industries. Applications of horizontal surfaces can also be found in various industrial exchanger systems. One of the fundamental problems of the engineering processes is the improvement in thermal systems during the convection in any fluid. The second law analysis is one of the best tools for improving the performance of the engineering processes. It investigates the irreversibility due to fluid flow and heat transfer in terms of the entropy generation rate. Bejan (1979, 1984, 1996) employed this method in many convection problems and later on several other investigators used this method successfully in different heat transfer problems.

Saouli and Saouli (2004) performed the second law analysis for Newtonian fluids along an inclined heated plate, whereas Saouli and Saouli (2009) and Gorla and Pratt (2007) performed the same analysis for non-Newtonian fluids with and without viscous dissipation effects. They considered the upper surface of the liquid film to be free and adiabatic and the lower wall fixed with constant heat flux. Their results show that the entropy generation number and the irreversibility ratio decrease in the transverse direction and increase as the viscous dissipation parameter increases. Recently, Saouli et al. (2006) investigated the entropy generation in a laminar, gravity-driven conducting liquid film along an

*To whom correspondence should be addressed 
inclined heated plate in the presence of a transverse magnetic field.

In this study we investigated the second law analysis of heat transfer and fluid flow due to free convection of non-Newtonian fluids over a horizontal plate with prescribed surface heat flux. The velocity and temperature distributions are determined by solving the Darcy and energy equations subject to an appropriate set of boundary conditions. The dimensionless entropy generation rate, irreversibility ratio and the Bejan number were computed using numerical values of the velocity, temperature and their gradients. The dimensionless parameters governing the problem are the dimensionless temperature variation parameter, fluid behavior index, the dimensionless transverse and axial distances, and Rayleigh number.

\section{ANALYSIS}

Consider a steady free convection of a power-law fluid along an impermeable horizontal plate (Fig. 1) with prescribed wall heat flux $\mathrm{Q}(\mathrm{x})=\mathrm{Ax}^{\boldsymbol{\lambda}}$ embedded in a porous medium. It is assumed that the physical properties of the power-law fluids, except the density, are constant and that the Boussinesq approximation for the case of maximum density is valid.

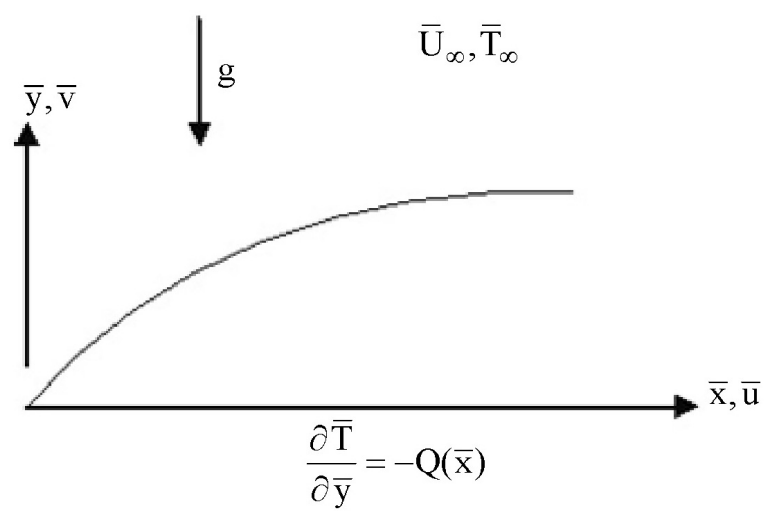

Figure 1: Flow model and coordinate system.

The ambient temperature is $\overline{\mathrm{T}}_{\infty}$ as shown in Fig. 1, where $\bar{x}$ and $\bar{y}$ are the Cartesian coordinates along the horizontal surface and normal to it, respectively. The velocity components are $\overline{\mathrm{u}}$ along and $\overline{\mathrm{v}}$ normal to the wall surface. The acceleration due to gravity $g$ acts vertically downwards. Assuming no heat friction and the thermal equilibrium between the fluid and the porous matrix, the governing equations in dimensional form, for this case, can be written as:

$$
\begin{aligned}
& \frac{\partial \overline{\mathrm{u}}}{\partial \overline{\mathrm{x}}}+\frac{\partial \overline{\mathrm{v}}}{\partial \overline{\mathrm{y}}}=0 \\
& \frac{\partial \overline{\mathrm{u}}^{\mathrm{n}}}{\partial \overline{\mathrm{x}}}=-\frac{\mathrm{K}^{*} \rho g \beta}{\mu} \frac{\partial \overline{\mathrm{T}}}{\partial \overline{\mathrm{x}}} \\
& \mathrm{u} \frac{\partial \overline{\mathrm{T}}}{\partial \overline{\mathrm{x}}}+\mathrm{v} \frac{\partial \overline{\mathrm{T}}}{\partial \overline{\mathrm{y}}}=\alpha \frac{\partial^{2} \overline{\mathrm{T}}}{\partial \overline{\mathrm{y}}^{2}}
\end{aligned}
$$

with boundary conditions:

$$
\begin{aligned}
& \bar{y}=0: \bar{v}=0, k \frac{\partial \bar{T}}{\partial \bar{y}}=-Q(\bar{x}) \\
& \bar{y} \rightarrow \infty: \bar{u}=0, \bar{T} \rightarrow \bar{T}_{\infty}
\end{aligned}
$$

where the bars denote dimensional quantities. Using the following non-dimensional variables

$$
\begin{aligned}
& \mathrm{x}=\frac{\overline{\mathrm{x}}}{\mathrm{L}}, \mathrm{y}=\frac{\overline{\mathrm{y} R \mathrm{a}^{1 / 3}}}{\mathrm{~L}}, \mathrm{u}=\frac{\overline{\mathrm{u}} \mathrm{L}}{\alpha \mathrm{Ra}^{2 / 3}} \\
& \mathrm{v}=\frac{\overline{\mathrm{u}} \mathrm{L}}{\alpha \mathrm{Ra}^{2 / 3}}, \overline{\mathrm{T}}=\mathrm{k} \frac{\overline{\mathrm{T}}-\overline{\mathrm{T}}_{\infty}}{\mathrm{q}_{\mathrm{o}} \mathrm{L}}, \mathrm{Q}=\frac{\mathrm{q}_{\mathrm{w}}}{\mathrm{q}_{\mathrm{o}} \mathrm{Ra}^{1 / 3}}
\end{aligned}
$$

where $\mathrm{q}_{0}$ is the characteristic heat flux, $\mathrm{Ra}$ is the modified Rayleigh number, which is defined by:

$R a=\left(\frac{L}{\alpha}\right)^{3 n /(2 n+1)}\left(\frac{\rho K^{*} g \beta q_{o} L}{k \mu}\right)^{3 /(2 n+1)}$

we get the following governing equations in dimensionless form:

$$
\frac{\partial \mathrm{u}}{\partial \mathrm{x}}+\frac{\partial \mathrm{v}}{\partial \mathrm{y}}=0
$$

$\frac{\partial u^{n}}{\partial y}=-\frac{\partial T}{\partial x}$

$\mathrm{u} \frac{\partial \mathrm{T}}{\partial \mathrm{x}}+\mathrm{v} \frac{\partial \mathrm{T}}{\partial \mathrm{y}}=\alpha \frac{\partial^{2} \mathrm{~T}}{\partial \mathrm{y}^{2}}$

with dimensionless boundary conditions:

$$
\begin{aligned}
& \mathrm{y}=0: \mathrm{v}=0, \mathrm{k} \frac{\partial \mathrm{T}}{\partial \mathrm{y}}=-\mathrm{Q}(\mathrm{x}) \\
& \mathrm{y} \rightarrow \infty: \overline{\mathrm{u}}=\mathrm{U}_{\infty}, \mathrm{T} \rightarrow \mathrm{T}_{\infty}
\end{aligned}
$$


Using the following transformations

$$
\begin{aligned}
& \eta=x^{(\lambda-n-1) / 2(n+1)} y \\
& \psi=x^{(\lambda+n+1) / 2(n+1)} f(\eta) \\
& T=x^{(2 n \lambda+\lambda+n+1) / 2(n+1)} \theta(\eta)
\end{aligned}
$$

we get:

$$
\begin{aligned}
& u=x^{\lambda /(n+1)} f^{\prime}(\eta) v=-x^{(\lambda-v-1) / 2(n+1)} \\
& \left\{\left(\frac{\lambda+n+1}{2(n+1)}\right) f(\eta)+\left(\frac{\lambda+n+1}{2(n+1)}\right) \eta f(\eta) f^{\prime}(\eta)\right\}
\end{aligned}
$$

and the governing equations reduce to:

$$
\begin{aligned}
& \mathrm{n}\left(\mathrm{f}^{\prime}\right)^{\mathrm{n}-1} \mathrm{f}^{\prime \prime}+ \\
& \left\{\frac{2 \mathrm{n} \lambda+\lambda+\mathrm{n}+1}{2(\mathrm{n}+1)}\right\} \theta+\left\{\frac{\lambda-\mathrm{n}-1}{2(\mathrm{n}+1)}\right\} \eta \theta^{\prime}=0 \\
& \theta^{\prime \prime}+\left\{\frac{\lambda+\mathrm{n}+1}{2(\mathrm{n}+1)}\right\} \mathrm{f} \theta^{\prime}-\left\{\frac{2 \mathrm{n} \lambda+\lambda+\mathrm{n}+1}{2(\mathrm{n}+1)}\right\} \mathrm{f}^{\prime} \theta=0
\end{aligned}
$$

with reduced boundary conditions:

$$
\begin{aligned}
& \mathrm{f}(0)=0, \theta^{\prime}(0)=-1 \\
& \mathrm{f}(\infty)=0, \theta(\infty)=0
\end{aligned}
$$

where primes denote the derivatives w. r. t. $\eta$. Equations (13) and (14) were solved numerically for the velocity and temperature profiles and gradients using an implicit finite difference method, where a step size of $\Delta \eta=0.001$ was used in all the calculations. These gradients are used in the calculations of dimensionless entropy generation rates. It is important to note that, for $\lambda=0$, Equations (13) and (14) reduce to the uniform wall flux case.

\section{Entropy Generation Rate}

Following Bejan [1], the local rate of entropy generation in convective heat transfer from a powerlaw fluid can be written as:

$$
\mathrm{S}_{\mathrm{g}}^{\prime \prime \prime}=\frac{\mathrm{k}}{\mathrm{T}_{\mathrm{o}}^{2}}\left[\left(\frac{\partial \overline{\mathrm{T}}}{\partial \overline{\mathrm{x}}}\right)^{2}+\left(\frac{\partial \overline{\mathrm{T}}}{\partial \overline{\mathrm{y}}}\right)^{2}\right]+\frac{\mu}{\mathrm{T}_{\mathrm{o}}}\left(\frac{\partial \overline{\mathrm{u}}}{\partial \overline{\mathrm{y}}}\right)^{\mathrm{n}+1}
$$

where

$$
\begin{aligned}
& \frac{\partial \overline{\mathrm{T}}}{\partial \overline{\mathrm{x}}}=\left(\frac{\mathrm{q}_{\mathrm{o}}}{\mathrm{k}}\right) \frac{\partial \mathrm{T}}{\partial \mathrm{x}}=\left(\frac{\mathrm{q}_{\mathrm{o}}}{\mathrm{k}}\right) \mathrm{x}^{\frac{2 \mathrm{n} \lambda+\lambda-\mathrm{n}-1}{2(\mathrm{n}+1)}} \\
& \left\{\frac{2 \mathrm{n} \lambda+\lambda+\mathrm{n}+1}{2(\mathrm{n}+1)} \theta+\frac{\lambda-\mathrm{n}-1}{2(\mathrm{n}+1)} \eta \theta^{\prime}\right\} \\
& \frac{\partial \overline{\mathrm{T}}}{\partial \overline{\mathrm{y}}}=\frac{\mathrm{q}_{\mathrm{o}} \mathrm{Ra}^{1 / 3}}{\mathrm{~L}} \frac{\partial \mathrm{T}}{\partial \mathrm{y}}=\frac{\mathrm{q}_{\mathrm{o}}}{\mathrm{k}} \mathrm{Ra}^{1 / 3} \mathrm{x}^{(\lambda-\mathrm{n}-1) / 2(\mathrm{n}+1)} \theta^{\prime} \\
& \frac{\partial \overline{\mathrm{u}}}{\partial \overline{\mathrm{y}}}=\frac{\partial\left(\mathrm{u} \alpha \mathrm{Ra}^{2 / 3} / \mathrm{L}\right)}{\partial(\mathrm{yLRa}}=\left(\frac{\alpha}{\mathrm{L}^{2}} \cdot \mathrm{Ra}\right) \frac{\partial \mathrm{u}}{\partial \mathrm{y}}= \\
& \left(\frac{\alpha}{\mathrm{L}^{2}} \cdot \mathrm{Ra}^{2 / 3}\right) \cdot \mathrm{x}^{(3 \lambda-\mathrm{n}-1) / 2(\mathrm{n}+1)} \mathrm{f}^{\prime \prime}
\end{aligned}
$$

Using Eqs. (17) and (18) in Eq. (16), we get dimensionless local rate of entropy generation:

$\mathrm{N}_{\mathrm{s}}=\mathrm{N}_{\mathrm{C}}+\mathrm{N}_{\mathrm{Y}}+\mathrm{N}_{\mathrm{F}}$

where:

$\mathrm{N}_{\mathrm{s}}=\frac{\mathrm{S}_{\mathrm{g}}^{\prime \prime \prime}}{\mathrm{q}_{\mathrm{o}}^{2} / \mathrm{kT}_{\mathrm{o}}^{2}}$

$\mathrm{N}_{\mathrm{c}}=\left[\mathrm{x}^{(2 \mathrm{n} \lambda+\lambda-\mathrm{n}-1) / 2(\mathrm{n}+1)}\left\{\begin{array}{c}\frac{2 \mathrm{n} \lambda+\lambda+\mathrm{n}+1}{2(\mathrm{n}+1)} \cdot \theta+ \\ \frac{\lambda-\mathrm{n}-1}{2(\mathrm{n}+1)} \eta \theta^{\prime}\end{array}\right\}\right]^{2}$

$\mathrm{N}_{\mathrm{Y}}=\mathrm{Ra}^{2 / 3} \quad\left[\mathrm{x}^{(\lambda-\mathrm{n}-1) / 2(\mathrm{n}+1)} \cdot \theta^{\prime}\right]^{2}$

$N_{F}=G \cdot R a^{n+1}\left[x^{(3 \lambda-n-1) / 2(n+1)} \cdot f^{\prime \prime}\right]^{n+1}$

with:

$\mathrm{G}=\frac{\mu \mathrm{kT} \mathrm{T}_{\mathrm{o}}^{2}}{\mathrm{q}_{\mathrm{o}}^{2}}\left(\frac{\alpha}{\mathrm{L}^{2}}\right)^{\mathrm{n}+1}$

where $T_{0}$ is the reference temperature, $N_{C}$ is the entropy generation in the axial direction, $\mathrm{N}_{\mathrm{Y}}$ is the entropy generation in the normal direction to the horizontal surface, $\mathrm{N}_{\mathrm{F}}$ is the entropy generation due 
to fluid friction and $G$ is the modified duty parameter.

The irreversibility ratio $\Phi$ is defined by Bejan (1984) as the ratio of the entropy generation due to the fluid friction $\mathrm{N}_{\mathrm{F}}$ to the total entropy generation due to heat transfer $\left(\mathrm{N}_{\mathrm{C}}+\mathrm{N}_{\mathrm{Y}}\right)$, i.e.

$$
\varphi=\frac{\mathrm{N}_{\mathrm{F}}}{\mathrm{N}_{\mathrm{C}}+\mathrm{N}_{\mathrm{Y}}}
$$

It is important to note that when $\varphi=1$, both the heat transfer and fluid friction irreversibilities make the same contribution to generating entropy. But, when $\varphi>1$, fluid friction dominates and, when $\varphi<1$, heat transfer dominates. Due to importance of the contribution of entropy generation due to heat transfer, Paoletti et al. (1989) defined the Bejan number $(\mathrm{Be})$ as the ratio of the entropy generation due to heat transfer to the total entropy generation, i.e.

$\mathrm{Be}=\frac{\mathrm{N}_{\mathrm{C}}+\mathrm{N}_{\mathrm{Y}}}{\mathrm{N}_{\mathrm{C}}+\mathrm{N}_{\mathrm{Y}}+\mathrm{N}_{\mathrm{F}}}=\frac{1}{1+\varphi}$

where the Bejan number ranges from 0 to 1 . When the heat transfer irreversibility dominates, $\mathrm{Be}=1$, when the irreversibility is dominated by fluid friction, $\mathrm{Be}=0$, and when the heat transfer and fluid friction entropy generation rates are equal $\mathrm{Be}=1 / 2$.

\section{RESULTS AND DISCUSSION}

Entropy generation rates due to heat transfer and fluid flow in free convection of Newtonian and nonNewtonian fluids over a horizontal plate with prescribed surface heat flux are analyzed graphically. Velocity and temperature fields are obtained by solving Equations (14) and (15) numerically with boundary conditions (16) using an implicit finite difference method under the similarity assumption. In order to validate the accuracy of the numerical results for dimensionless entropy generation rates and Bejan numbers, dimensionless heat transfer coefficients for free convection along a horizontal plate with prescribed wall heat flux are compared for a Newtonian fluid $(\mathrm{n}=1)$ with those of Gorla and Kumari (2003), and Aldoss et al. (1994). The differences between their results and the present results is less than 0.0098 . Therefore, our results are highly accurate. This comparison is presented in Table 1.

Table 1: Comparison of dimensionless heat transfer coefficients for free convection along a horizontal plate

\begin{tabular}{|l|c|c|c|}
\hline $\boldsymbol{\lambda}$ & $\begin{array}{c}\text { Gorla and Kumari } \\
\mathbf{( 2 0 0 3 )}\end{array}$ & $\begin{array}{c}\text { Aldoss } \text { et al. } \\
\mathbf{( 1 9 9 4 )}\end{array}$ & $\begin{array}{c}\text { Present } \\
\text { Results }\end{array}$ \\
\hline 0 & 0.85835 & 0.8589 & 0.8582 \\
0.5 & 1.02444 & 1.0248 & 1.0239 \\
1 & 1.1651 & 1.165 & 1.1587 \\
1.5 & 1.28963 & 1.2896 & 1.2869 \\
2 & 1.40317 & 1.4032 & 1.3987 \\
\hline
\end{tabular}

The dimensionless entropy generation rates for a Newtonian fluid $(\mathrm{n}=1)$, a pseudoplastic fluid $(\mathrm{n}=0.5)$, and a dilatant fluid $(\mathrm{n}=1.5)$ over a horizontal plate in porous media are plotted in Figures 2-4 respectively. In each case, the effects of the viscous dissipation parameter $\mathrm{G}$ and the Rayleigh number $\mathrm{Ra}$ on the dimensionless entropy generation rate Ns are shown for different values of the temperature variation parameter $\lambda$. As expected, the entropy generation rate for Newtonian fluids is maximum at the plate surface due to maximum heat transfer and friction. However, there is no contribution to the entropy generation rate at the free surface, due to zero velocity and temperature gradients (Figure 2). For a Newtonian fluid, the entropy generation rates increase with the viscous dissipation parameter due to the increase in friction and they decrease with the increase in the flux variation parameter $\lambda$ (Fig. 2(a). Figure 2(b) shows that the entropy generation rates increase with the increase in the Rayleigh number Ra. The variation of the dimensionless entropy generation rate with the transverse distance $\eta$ is shown in Figure 3 for a pseudoplastic fluid $(n=0.5)$. Like Newtonian fluids, the entropy generation rates increase with the increase in viscous dissipation $G$ and the Rayleigh number Ra. This is due to the decrease in heat transfer along the axial distance. Again, the dimensionless entropy generation rates are lower for higher values of the flux variation parameter $\lambda$. For dilatant fluids $(n=1.5)$, the entropy generation rates decrease initially with the transverse distance and then increase asymptotically for all parameters as shown in Figures 4(a) and 4(b), due to the increase in heat transfer and fluid friction irreversibilities. For this reason, dilatant fluids are not recommended for free convection along a horizontal plate under uniform surface temperature. 


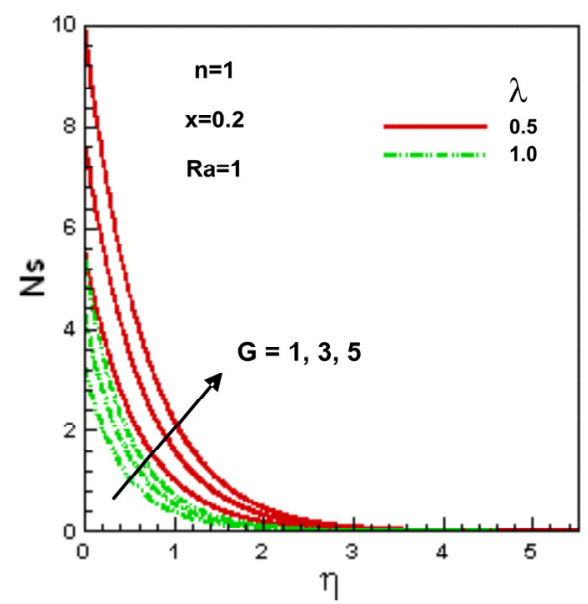

(a)

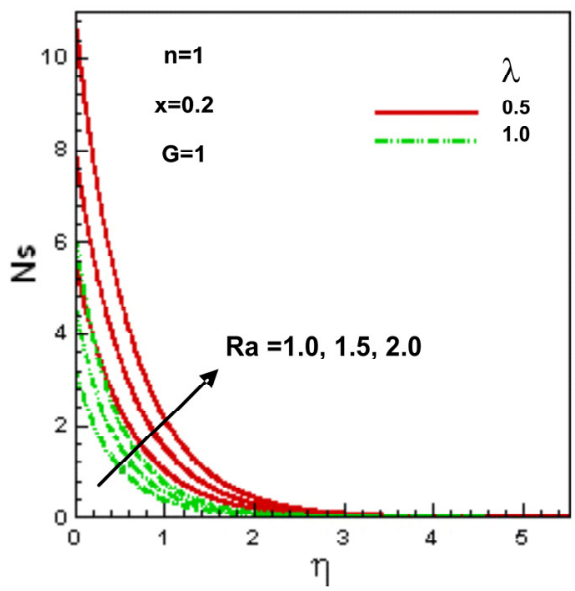

(b)

Figure 2: Effect of $\lambda, G$ and Ra on the dimensionless entropy generation rate Ns for Newtonian fluids.

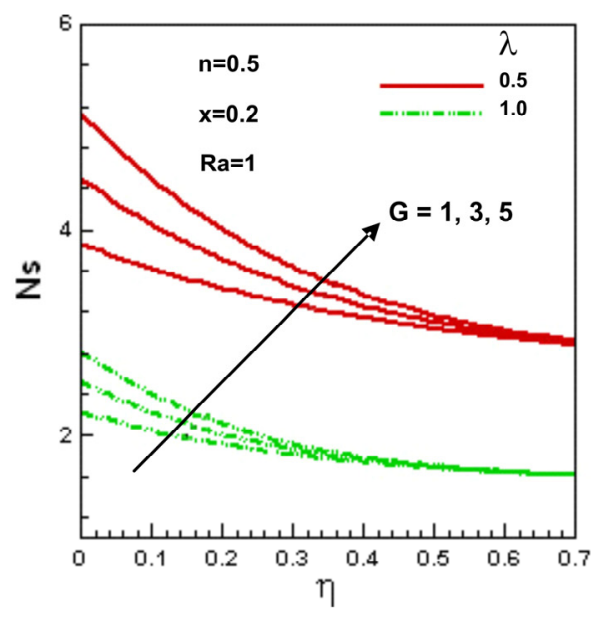

(a)

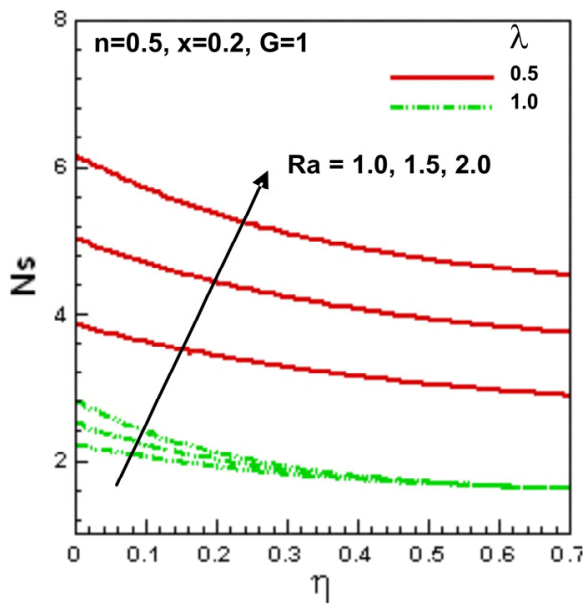

(b)

Figure 3: Effect of $\lambda, \mathrm{G}$ and Ra on the dimensionless entropy generation rate Ns for pseudo-plastic fluids.

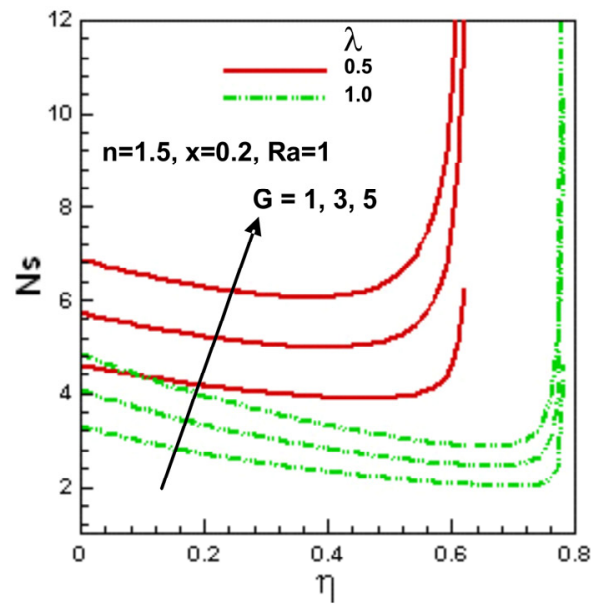

(a)

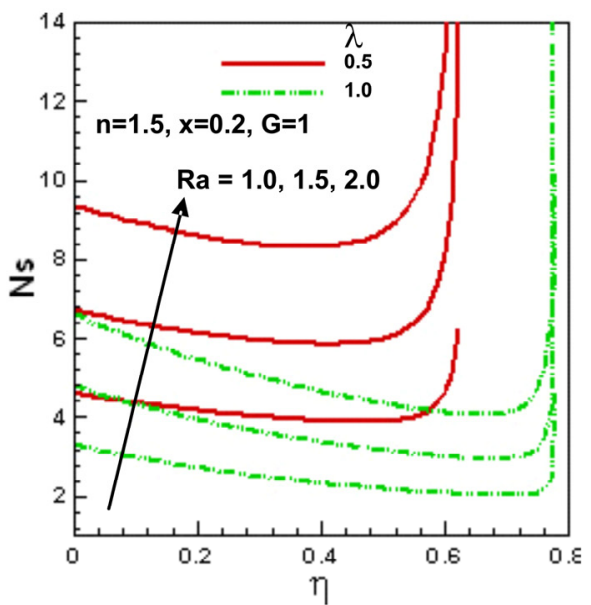

(b)

Figure 4: Effect of $\lambda, \mathrm{G}$ and $\mathrm{Ra}$ on the dimensionless entropy generation rate Ns for dilatant fluids. 
The variation of the Bejan number with the transverse distance for Newtonian, pseudoplastic and dilatant fluids is shown in Figures 5-7, respectively. They show the effects of the viscous dissipation parameter and Rayleigh numbers on the Bejan number. It is clear from Figure 5 that, for the Newtonian fluids, the Bejan number is a decreasing function of the dimensionless transverse distance and it decreases with the increase in the viscous dissipation parameter and the Rayleigh number, as shown in Figures 5(a) and 5(b), whereas the Bejan number increases with the increase in the temperature variation parameter in both cases. The Bejan number is higher at the plate surface due to higher heat transfer irreversibility. Unlike Newtonian fluids, the Bejan number for pseudoplastic fluids is an increasing function of the dimensionless transverse distance and it decreases with the increase in the viscous dissipation number and the Rayleigh

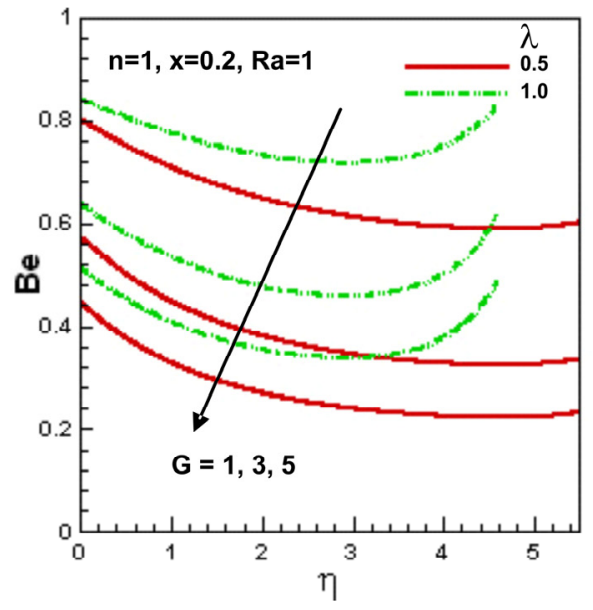

(a) number, as shown in Figures 6(a) and 6(b). The Bejan number increases with the increase in the flux variation parameter in both cases. This is due to the fact that the heat transfer irreversibility increases with the increase in the flux variation parameter. In the case of dilatant fluids, the heat transfer irreversibility is higher at the plate surface and remains constant initially, then fluid friction irreversibility dominates and, as a result, the Bejan number decreases with the transverse distance, as shown in Figures 7(a) and 7(b). Due to the nature and rheological properties of dilatant fluids, the heat transfer irreversibilities decrease with the increase in viscous dissipation and Rayleigh numbers and, as a result, the Bejan number decreases. However, with the increase in the flux variation and axial distance, heat transfer increases and, as a result, the Bejan number increases, as shown in Figures 7(a) and 7(b), respectively.

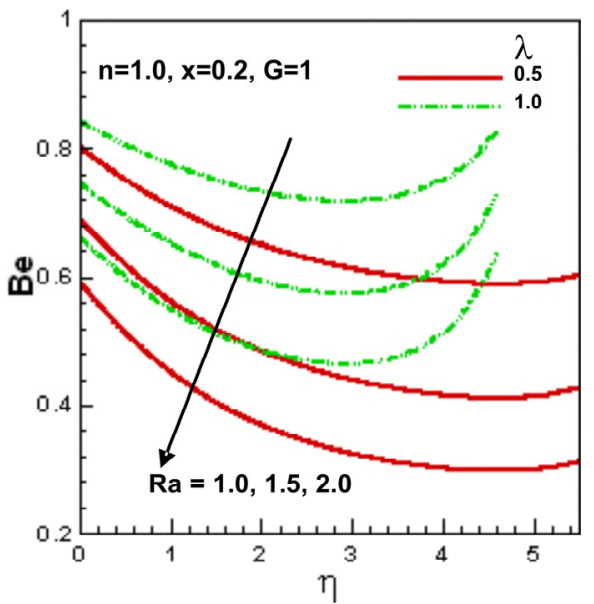

(b)

Figure 5: Effect of $\lambda, \mathrm{G}$ and $\mathrm{Ra}$ on the Bejan number Be for Newtonian fluids.

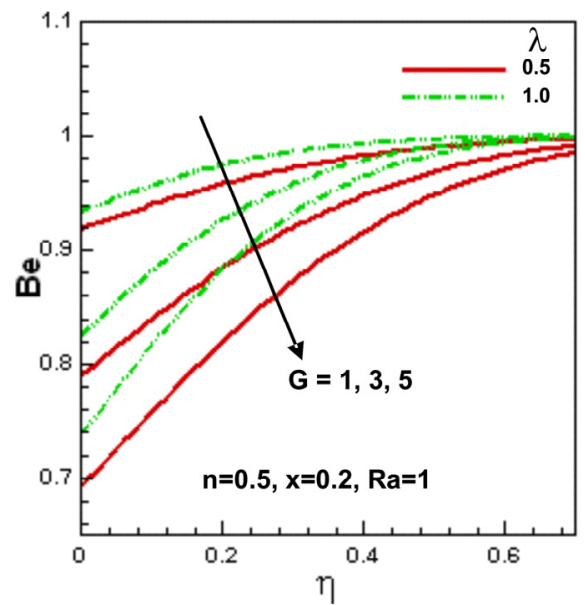

(a)

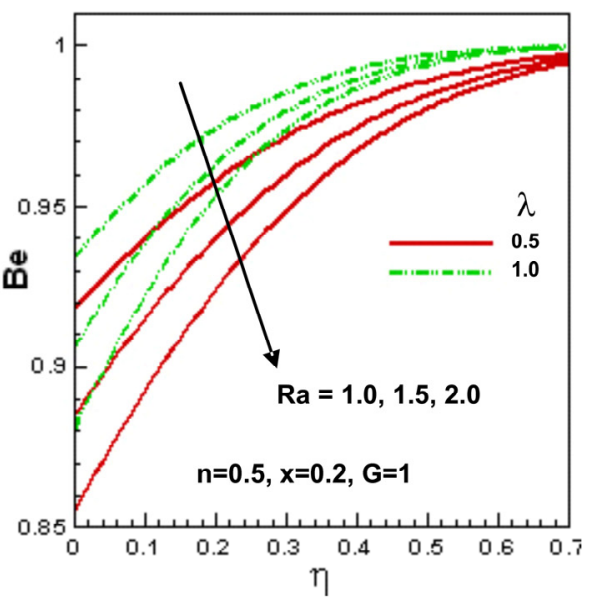

(b)

Figure 6: Effect of $\lambda, \mathrm{G}$ and Ra on the Bejan number Be for pseudo-plastic fluids. 


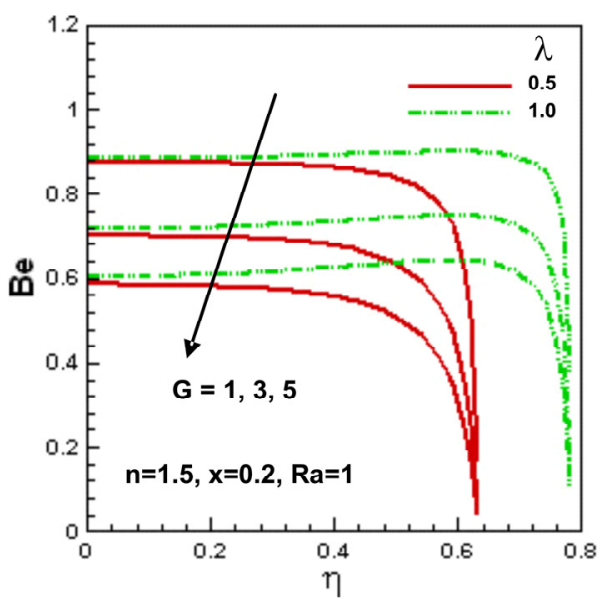

(a)

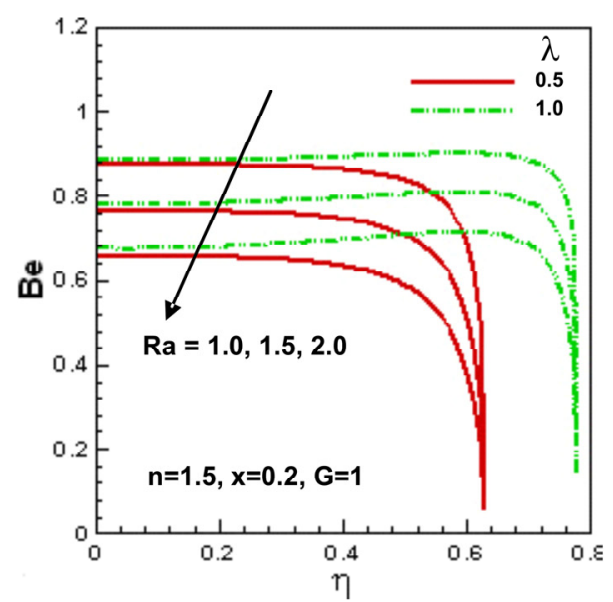

(b)

Figure 7: Effect of $\lambda, G$ and $\mathrm{Ra}$ on the Bejan number Be for dilatant fluids.

\section{CONCLUSIONS}

Second law analysis of heat transfer and fluid flow due to free convection of Newtonian and nonNewtonian fluids over a horizontal plate is performed for prescribed surface flux boundary conditions. Velocity and temperature fields are obtained numerically using an implicit finite difference method under the similarity assumption. These results are then used to determine the dimensionless entropy generation rates and Bejan numbers. It is observed that the entropy generation rate and Bejan number depend upon the viscous dissipation parameter $G$, the temperature variation parameter $\lambda$, the Rayleigh number $\mathrm{Ra}$, the fluid behaviour index $\mathrm{n}$, and the transverse and axial distances.

\section{NOMENCLATURE}

Be Bejan number

f dimensionless stream

function

G modified duty parameter

g acceleration due to gravity

$\mathrm{K}^{*} \quad$ permeability of the porous medium,

$\mathrm{N}_{\mathrm{C}} \quad$ dimensionless entropy generation rate in the axial direction

$\mathrm{N}_{\mathrm{Y}}$ dimensionless entropy generation rate in the normal direction
$\mathrm{N}_{\mathrm{F}} \quad$ dimensionless entropy generation rate due to fluid friction
$\mathrm{N}_{\mathrm{s}} \quad$ dimensionless local entropy generation rate
$\mathrm{Nu} \quad$ Nusselt number
n power-law index
Ra modified Rayleigh number
$\mathrm{T}$ dimensionless temperature
$\mathrm{u}, \mathrm{v} \quad$ velocity components in the $\mathrm{x}$ - and $\mathrm{y}$ - directions
$\mathrm{x}, \mathrm{y} \quad$ coordinates along and normal to the surface

\section{Subscripts}

$\begin{array}{ll}\infty & \text { free stream conditions } \\ 0 & \text { reference temperature } \\ \mathrm{n} & \begin{array}{l}\text { free convection dominated } \\ \text { regime }\end{array} \\ \mathrm{w} & \text { wall }\end{array}$

\section{Greek Symbols}

$\alpha$ thermal diffusivity of $\quad \mathrm{m}^{2} / \mathrm{s}$

$\begin{array}{rlll}\mathrm{m} / \mathrm{s}^{2} & \alpha & \begin{array}{l}\text { thermal diffusivity of } \\ \text { porous medium }\end{array} & \mathrm{m}^{2} / \mathrm{s} \\ \mathrm{m}^{2} & \beta & \begin{array}{l}{ }^{\circ} \mathrm{C}^{-2} \\ \text { thermal expansion }\end{array}\end{array}$

Newtonian fluid

$\lambda$ temperature variation

parameter

$\mu \quad$ absolute viscosity

$v \quad$ kinematic viscosity $\mathrm{kg} / \mathrm{ms}$ $\mathrm{m}^{2} / \mathrm{s}$ 
$\Phi \quad$ irreversibility ratio

\section{REFERENCES}

Aldoss, T. K., Chen, T. S. and Armaly, B. F., Mixed convection over nonisothermal horizontal surfaces in a porous medium: The entire regime. Numerical Heat Transfer, 25, 685 (1994).

Bejan, A., A study of entropy generation in fundamental convective heat transfer. J. Heat Transfer, 101, 718 (1979).

Bejan, A., Convection Heat Transfer. Wiley, New York (1984).

Bejan, A., Entropy Generation Minimization. CRC Press, Boca Raton, New York (1996).

Gorla, R. S. R. and Kumari, M., Free convection in Non-Newtonian fluids along a horizontal plate in a porous medium. Heat and Mass Transfer, 39, $101(2003)$.

$\mathrm{kg} / \mathrm{m}^{3}$
W. Khan and R. S. R. Gorla

Gorla, R. S. R. and Pratt, D. M., Second law analysis of a Non-Newtonian laminar falling liquid film along an inclined heated plate. Entropy, 9, 30 (2007).

Paoletti, S., Rispoli, F., Sciubba, E., Calculation of exergetic losses in compact heat exchanger passages. ASME AES, 10, 21 (1989).

Saouli, S., Saouli-Aboud, S, Second law analysis of laminar falling liquid film along an inclined heated plate. International Communications in Heat and Mass Transfer, 31, 879 (2004).

Saouli, A., Saouli, S., Settou, N., Meza, N., Thermodynamic analysis of gravity-driven liquid film along an inclined heated plate with hydromagnetic and viscous dissipation effects. Entropy, 8, 188 (2006).

Saouli, S. and Saouli, A., Second-law analysis of laminar Non-Newtonian gravity-driven liquid film along an inclined heated plate with viscous dissipation. Brazilian Journal of Chemical Engineering, 26, No. 2, 407 (2009). 